\title{
Reliability Measures of a Two-Unit Cold Standby Repairable System with Priority to Operation over Preventive Maintenance
}

\author{
Sonali Baweja \\ Department of mathematics \\ Manipal University Jaipur \\ Jaipur-303007, India
}

\author{
Ashish Kumar \\ Department of mathematics \\ Manipal University Jaipur \\ Jaipur-303007, India
}

\begin{abstract}
The main objective of present work is to carry out various important reliability measures of a two-unit cold standby repairable system with priority to operation over preventive maintenance by using regenerative points technique and semi-Markov process. A single repair facility is provided to conduct repair and maintenance of the system as and when required. All times distributions are exponential except the repair and preventive maintenance time distributions which are considered as general.
\end{abstract}

\section{General Terms}

Reliability Analysis

\section{Keywords}

Cold Standby System, Preventive Maintenance, Priority and Profit Function

\section{INTRODUCTION}

Malik [1] studied some stochastic models with priority to operation over repair with arrival time of the server and derived several measures of reliability for the system. Malik et al. [2] derived the expressions for economic analysis of repairable systems by using the concept of random appearance and disappearance of the server. In Ref. [1] and Ref. [2] the authors did not take the concepts of preventive maintenance after a maximum operation time and priority to operation over preventive maintenance.

Many papers [3-5] dealt with repairable standby systems subject to different repair policies and preventive maintenance, but they considered that the operating unit may go for preventive maintenance when other unit is also under repair or maintenance. Practically this is not true at all.

The goal of the present study is to discuss a two-unit cold standby repairable system with priority to operation over preventive maintenance. The following measures of reliability for the system are obtained:

- Transition probabilities

- Mean sojourn times

- Mean time to system failure

- Steady State Availability

- Busy period of the server due to repair

- Busy period of the server due to preventive maintenance

- Expected number of repairs

- Expected number of preventive maintenances

- Expected number of visits by the server

- $\quad$ Expected profit earned by the system in $(0, t)$.
2. MODEL DESCRIPTION

(i) Initially system consists of two identical units- one operative and other is kept as cold standby.

(ii) Both units have three modes- normal, under repair due to failure and under preventive maintenance.

(iii) The failure and maximum operation time distribution are exponentially distributed while repair and preventive maintenance times are distributed arbitrarily.

(iv) There is a single server who visits the system immediately as and when required.

(v) The switch devices, repairs and preventive maintenance are perfect.

(vi) All random variables are statistically independent.

\section{METHODOLOGY}

The system has been analyzed using well known semiMarkov process and regenerative point technique which are briefly described as:

\subsection{Markov Process:}

If $\{\mathrm{X}(\mathrm{t}), \mathrm{t} \in T\}$ is a stochastic process such that, given the value of $X(s)$, the value of $X(t), t>s$ do not depend on the values of $\mathrm{X}(\mathrm{u}), \mathrm{u}<\mathrm{s}$ Then the process $\{\mathrm{X}(\mathrm{t}), \mathrm{t} \in \mathrm{T}\}$ is a Markov process.

\subsection{Semi-Markov Process:}

A semi-Markov process is a stochastic process in which changes of state occur according to a Markov chain and in which the time interval between two successive transitions is a random variable, whose distribution may depend on the state from which the transition take place as well as on the state to which the next transition take place.

\subsection{Regenerative Process:}

Regenerative stochastic process was defined by Smith (1955) and has been crucial in the analysis of complex system. In this, we take time points at which the system history prior to the time points is irrelevant to the system conditions. These points are called regenerative points. Let $\mathrm{X}(\mathrm{t})$ be the state of the system of epoch. If $t_{1}, t_{2}, \ldots$ are the epochs at which the process probabilistically restarts, then these epochs are called regenerative epochs and the process $\left\{\mathrm{X}(\mathrm{t}), \mathrm{t}=\mathrm{t}_{1}, \mathrm{t}_{2} \ldots \ldots \ldots\right\}$ is called regenerative process. The state in which regenerative points occur is known as regenerative state. 


\section{NOTATIONS}

$\mathrm{O} \quad$ : The unit is operative and in normal mode.

Cs : The unit is in cold standby.

$\Lambda \quad$ : Constant failure rate of the unit.

$\alpha_{0} \quad$ : Constant rate of Maximum Operation Time.

$\mathrm{m}(\mathrm{t}) / \mathrm{M}(\mathrm{t}) \quad$ : pdf / cdf of preventive maintenance of the unit after maximum operation time.

Pm/PM : The unit is under preventive Maintenance/ under preventive maintenance continuously from previous state.

FUr/FUR : The unit is failed and is under repair / under repair continuously from previous state

FWr / : The unit is failed and is waiting for repair/ FWR waiting for repair from previous state

$\mathrm{g}(\mathrm{t}) / \mathrm{G}(\mathrm{t}) \quad$ : $\quad$ dff / cdf of repair time of the failed unit

$\mathrm{q}_{\mathrm{ij}}(\mathrm{t}) / \quad$ : pdf/cdf of direct transition time from a

$\mathrm{Q}_{\mathrm{ij}}(\mathrm{t}) \quad$ regenerative state $\mathrm{i}$ to a regenerative state $\mathrm{j}$ without visiting any other regenerative state.

$\mathrm{q}_{\mathrm{ij.k}}(\mathrm{t}) \quad /$ : pdf/cdf of first passage time from a $\mathrm{Q}_{\mathrm{ij.k}}(\mathrm{t}) \quad$ regenerative state $\mathrm{i}$ to a regenerative state $\mathrm{j}$ or to a failed state $\mathrm{j}$ visiting state $\mathrm{k}$ once in $(0, \mathrm{t}]$.

$\mathrm{M}_{\mathrm{i}}(\mathrm{t}) \quad$ : Probability that the system is up initially in state $\mathrm{S}_{\mathrm{i}} \boldsymbol{E} \mathrm{E}$ is up at time ' $\mathrm{t}$ ' without visiting to any other regenerative state.

$\mathrm{pdf} / \mathrm{cdf} \quad$ : Probability density function/ Cumulative density function

$\mathrm{W}_{\mathrm{i}}(\mathrm{t}) \quad$ : Probability that the server is busy in state $\mathrm{S}_{\mathrm{i}}$ up to time $t$ without making transition to any other regenerative state or returning to the same via one or more non regenerative states.

$\mathrm{m}_{\mathrm{ij}} \quad$ : Contribution to mean sojourn time in state $\mathrm{S}_{\mathrm{i}}$ when system transits directly to state $S_{j}\left(S_{i}, S_{j}\right.$ EE ) so that $\mu_{\mathrm{i}=} \boldsymbol{\Sigma}_{j} \mathrm{~m}_{\mathrm{ij}}$ where $\mathrm{m}_{\mathrm{ij}}=\int t$ $\mathrm{dQ}_{\mathrm{ij}}(\mathrm{t})=-\mathrm{q}_{\mathrm{ij}}{ }^{*}(0)$ and $\mu_{\mathrm{i}}$ is the mean sojourn time in state $S_{i} \in E$

$\mu_{i} \quad: \quad$ The mean Sojourn time in state $\mathrm{S}_{\mathrm{i}}$ this is given by

$$
\mu_{i}=E\left(T_{i}\right)=\int_{0}^{\infty} P\left(T_{i}>t\right) d t=\sum_{j} m_{i j}
$$

, where $T_{i}$ is the sojourn time in state $\mathrm{S}_{\mathrm{i}}$.

(S) / (C) : Symbol for Stieltjes convolution / Laplace convolution

$\sim$ / : Symbol for Laplace Stieltjes Transform (LST) / Laplace Transform (LT).

The system may be in one of the following states:

$S_{0}=(o, C s), \quad S_{1}=(o, F u r), \quad S_{2}=(o, P m)$,

$S_{3}=(P M, F w r)$ and $S_{4}=(F U R, F w r)$
Up states are: $S_{0}, S_{1}$ and $S_{2}$. Down States are: $S_{3}$ and $S_{4}$. Regenerative states: $S_{0}, S_{1}$ and $S_{2}$.Non-regenerative states: $S_{3}$ and $S_{4}$.

\section{TRANSITION PROBABILITIES AND MEAN SOJOURN TIMES}

Simple probabilistic considerations yield the following

expressions for the non-zero elements

$p_{i j}=Q_{i j}(\infty)=\int_{0}^{\infty} q_{i j}(t) d t$

$P_{01}=\frac{\gamma_{1}}{\gamma_{1}+\alpha_{0}} \quad, \quad P_{02}=\frac{\alpha_{0}}{\gamma_{1}+\alpha_{0}}, P_{14}=1-g^{*}\left(\gamma_{1}\right), \quad P_{10}=$ $g^{*}\left(\gamma_{1}\right), P_{23}=1-f^{*}\left(\gamma_{1}\right)$,

$P_{20}=f^{*}\left(\gamma_{1}\right), \quad P_{31}=1, \quad P_{41}=1, \quad P_{11.4}=\left[1-g^{*}\left(\gamma_{1}\right)\right]$,

$P_{21.3}=1-f^{*}\left(\gamma_{1}\right)$,

It can be easily verified that: $P_{01}+P_{02}=P_{14}+P_{10}=P_{23}+$ $P_{20}=P_{31}=P_{41}=P_{11.4}+P_{21.3}=1$

The mean sojourn times $\left(\mu_{\mathrm{i}}\right)$ of the state $\mathrm{S}_{\mathrm{i}}$ are

$\mu_{0}=\frac{1}{\alpha_{0}+\gamma_{1}}, \quad \mu_{1}=\frac{1}{\theta+\gamma_{1}}, \mu_{2}=\frac{1}{\alpha+\gamma_{1}}, \quad \mu_{1}^{\prime}=\frac{1}{\theta}, \quad \mu_{2}^{\prime}=\frac{1}{\alpha}$

\section{RELIABILITY AND MEAN TIME TO SYSTEM FAILURE (MTSF)}

Let $\varphi_{i}(t)$ be the cdf of first passage time from the regenerative state $S_{i}$ to a failed state. Regarding the failed state as absorbing state, we have the following recursive relations for $\varphi_{i}(t)$;

$\varphi_{i}(t)=\sum_{j} Q_{i, j}(t) \circledast \varphi_{j}(t)+\sum_{k} Q_{i, k}(t)$

Where $S_{j}$ is an un-failed regenerative state to which the given regenerative state $S_{i}$ can transit and $S_{k}$ is a failed state to which the state $S_{i}$ can transit directly. Taking LST of above relation (5) and solving for $\tilde{\phi}_{0}(s)$. We have

$\mathrm{R} *(\mathrm{~s})=\frac{1-\widetilde{\phi}_{0}(s)}{s}$

The reliability of the system model can be obtained by taking inverse Laplace transform of (6).

The mean time to system failure (MTSF) is given by

$\mathrm{MTSF}=\lim _{s \rightarrow o} \frac{1-\widetilde{\phi}_{0}(s)}{s}=\frac{N_{1}}{D_{1}}$ where

$N_{1}=p_{01} p_{14}+p_{02} p_{23}$ and $D_{1}=1-p_{01} p_{10}+p_{02} p_{20}$ 


\section{STEADY STATE AVAILABILITY}

Let $\mathrm{A}_{\mathrm{i}}(\mathrm{t})$ be the probability that the system is in up-state at instant 't' given that the system entered regenerative state $S_{i}$ at $t=0$. The recursive relations for $A_{i}(t)$ are given as

$$
A_{i}(t)=M_{i}(t)+\sum_{j} q_{i, j}^{(n)}(t) \subseteq A_{j}(t)
$$

where $S_{j}$ is any successive regenerative state to which the regenerative state $S_{i}$ can transit through $\mathrm{n}$ transitions. $\mathrm{M}_{\mathrm{i}}(\mathrm{t})$ is the probability that the system is up initially in state $S_{i} \in E$ up at time $\mathrm{t}$ without visiting to any other regenerative state, we have is

$$
\begin{aligned}
& M_{0}(t)=e^{-\left(\lambda+\alpha_{0}\right) t}, M_{1}(t)=e^{-\left(\lambda+\alpha_{0}\right) t \overline{F(t)}}, \\
& M_{2}(t)=e^{-\left(\lambda+\alpha_{0}\right) t \overline{H(t)}, M_{4}(t)=e^{-\left(\lambda+\alpha_{0}\right) t \overline{G(t)}}} \\
& M_{3}(t)=e^{-\left(\lambda+\alpha_{0}\right) t \overline{M(t)}}
\end{aligned}
$$

Taking LT of above relations (8) and solving for $A_{0}^{*}(s)$. The steady state availability is given by

$$
A_{0}(\infty)=\lim _{s \rightarrow 0} s A_{0}^{*}(s)=\frac{N_{2}}{D_{2}}, \text { where }
$$

$$
\begin{aligned}
& \mathrm{N}_{2}= \\
& \left(\mu_{o}\right)\left[1-p_{11.4}\right]+\left(\mu_{1}\right)\left[p_{01}+p_{02} p_{21.3}\right]+\left(\mu_{2}\right)\left[p_{02}\left(1-p_{11.4}\right)\right]
\end{aligned}
$$

And

$\mathrm{D}_{2}=$

$\left(\mu_{0}\right)\left[1-p_{11.4}\right]+\left(\mu_{1}^{\prime}\right)\left[p_{01}+p_{01} p_{21.3}\right]+\left(\mu_{2}^{\prime}\right)\left[p_{02}\left(1-p_{11.4}\right)\right]$

\section{BUSY PERIOD ANALYSIS OF THE SERVER}

\subsection{Due to Preventive Maintenance (PM)}

Let $B_{i}{ }^{P}(t)$ be the probability that the server is busy in preventive maintenance of the system (unit) at an instant ' $t$ ' given that the system entered state $S_{i}$ at $\mathrm{t}=0$. The recursive relations for $B_{i}^{P}(t)$ are as follows:

$B_{i}^{p}(t)=W_{i}(t)+\sum_{j} q_{i, j}^{(n)}(t) \Subset B_{j}^{p}(t)$

Where $S_{j}$ is any successive regenerative state to which the regenerative state $S_{i}$ can transit through $\mathrm{n}$ transitions. $\mathrm{W}_{\mathrm{i}}(\mathrm{t})$ be the probability that the server is busy in state $S_{i}$ due to preventive maintenance up to time $t$ without making any transition to any other regenerative state or returning to the same via one or more non-regenerative states and so

$$
W_{1}(t)=e^{-\left(\lambda+\alpha_{0}\right) t} \bar{F}(t)+\left(\alpha_{0} e^{-\left(\lambda+\alpha_{0}\right) t} \mathrm{C} 1\right) \overline{\mathrm{F}}(t)+\left(\lambda e^{-\left(\lambda+\alpha_{0}\right) t} \mathrm{C} 1\right) \bar{F}(t)
$$

\subsection{Due to Repair}

Let $B_{i}^{R}(t)$ be the probability that the server is busy in repairing the unit due to failure at an instant ' $t$ ' given that the system entered state $S_{i}$ at $\mathrm{t}=0$. The recursive relations for $B_{i}^{R}(t) \quad$ are
$B_{i}^{R}(t)=W_{i}(t)+\sum_{j} q_{i, j}^{(n)}(t) \subseteq B_{j}^{R}(t)$ follows:

where $S_{j}$ is any successive regenerative state to which the regenerative state $S_{i}$ can transit through n transitions. $\mathrm{W}_{\mathrm{i}}(\mathrm{t})$ be the probability that the server is busy in state $S_{i}$ due to repair of the unit up to time $t$ without making any transition to any other regenerative state or returning to the same via one or more non-regenerative states and so

$W_{2}=e^{-\left(\lambda+\alpha_{0}\right) t} \bar{G}(t)+\left(\alpha_{0} e^{-\left(\lambda+\alpha_{0}\right) t}(1) \bar{G}(t)+\left(\lambda e^{-\left(\lambda+\alpha_{0}\right) t} \subseteq 1\right) \bar{G}(t)\right.$

Taking LT of above relations and solving for $B_{0}^{*^{P}}$ (s) \& $B_{0}{ }^{*}$ (s). The time for which server is busy due to preventive maintenance and repair is

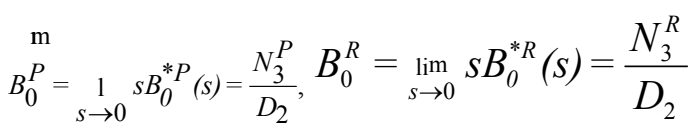

Where $N_{3}^{P}=W_{2}^{*}(0)\left[p_{02}\left(1-p_{11.4}\right)\right.$,

$N_{3}^{R}=W_{1}^{*}(0)\left[p_{01}+p_{02} p_{21.3}\right]$ and $\quad D_{2}$ is already defined.

\section{EXPECTED NUMBER OF PREVENTIVE MAINTENANCES}

Let $R_{i}^{p}(t)$ be the expected number of preventive maintenances conducted by the server in $(0, t]$ given that the system entered the regenerative state $S_{i}$ at $\mathrm{t}=0$. The recursive relations for $R_{i}^{p}(t)$ are given as

$$
R_{i}^{p}(t)=\sum_{j} Q_{i, j}^{(n)}(t) \circledast\left[\delta_{j}+R_{j}^{p}(t)\right]
$$

where $S_{j}$ is any regenerative state to which the given regenerative state $S_{i}$ transits and $\delta j=1$, if $S_{j}$ is the regenerative state where the server does job afresh, otherwise $\delta j=0$. Taking LST of relations (11) and solving for $\tilde{R}_{0}^{p}(s)$. The expected numbers of preventive maintenances per unit time are given by 
$R_{0}^{p}(\infty)=\lim _{s \rightarrow 0} s \tilde{R}_{0}^{p}(s)=\frac{N_{4}^{p}}{D_{2}}$

Where $N_{4}^{p}=\left[p_{02}-p_{11.4} p_{02}\right]$ and $D_{2}$ is already mentioned.

\section{EXPECTED NUMBERS OF REPAIRS}

Let $R_{i}^{r}(t)$ be the expected number of repairs by the server in $(0, \mathrm{t}]$ given that the system entered the regenerative state $S_{i}$ at $\mathrm{t}=0$. The recursive relations for $R_{i}^{r}(t)$ are given as

$$
R_{i}^{r}(t)=\sum_{j} Q_{i, j}^{(n)}(t) \circledast\left[\delta_{j}+R_{j}^{r}(t)\right]
$$

Where $S_{j}$ is any regenerative state to which the given regenerative state $S_{i}$ transits and $\delta j=1$, if $S_{j}$ is the regenerative state where the server does job afresh, otherwise $\delta j=0$. Taking LST of relations (12) and solving for $\tilde{R}_{0}^{r}(S)$ . The expected numbers of repairs per unit time are given by

$$
R_{0}^{r}(\infty)=\lim _{s \rightarrow 0} s \tilde{R}_{0}^{r}(s)=\frac{N_{4}^{r}}{D_{2}}
$$

Where $N_{4}^{r}=\left[p_{01}+p_{02} p_{21.3}\right]$ and $\mathrm{D}_{2}$ is already mentioned.

\section{EXPECTED NUMBERS OF VISITS BY THE SERVER}

Let $N_{i}(t)$ be the expected number of visits by the server in $(0, \mathrm{t}]$ given that the system entered the regenerative state $S_{i}$ at $\mathrm{t}=0$. The recursive relations for $N_{i}(t)$ are given as ;

$$
N_{i}(t)=\sum_{j} Q_{i, j}^{(n)}(t){ }^{\circledR}\left[\delta_{j}+N_{i}(t)\right]
$$

where $S_{j}$ is any regenerative state to which the given regenerative state $S_{i}$ transits and $\delta j=1$, if $j$ is the regenerative state where the server does job afresh, otherwise $\delta j=0$. Taking LST of relations (13) and solving for $N_{0}(s)$. The expected numbers of replacements per unit time are given by $N_{0}(\infty)=\lim _{s \rightarrow 0} s \tilde{N}_{0}(s)=\frac{N_{5}}{D_{2}}$

where $N_{0}=\left[1-p_{11.4}\right]$ and $\mathrm{D}_{2}$ is already mentioned.

\section{PROFIT ANALYSIS}

The profit incurred to the system model in steady state can be obtained as

$P=K_{0} A_{0}-K_{1} B_{0}^{R}-K_{2} B_{0}^{P}-K_{3} R_{0}^{R}-K_{4} R_{0}^{P}-K_{5} N_{0}$ $\mathrm{K} 0=$ Revenue per unit up-time of the system

$\mathrm{K} 1=$ Cost per unit time for which server is busy due to repair

$\mathrm{K} 2=$ Cost per unit time for which server is busy due to preventive maintenance

$\mathrm{K} 3=$ Cost per unit time repair of the unit

$\mathrm{K} 4=$ Cost per unit time preventive maintenance of the unit

$\mathrm{K} 5=$ Cost per unit time visit by the server

\section{CONCLUSION}

The numerical results for some reliability measures are obtained in tables 1-3 by considering the particular case $g(t)=\theta e^{-\theta t}$ and $f(t)=\alpha e^{-\alpha t}$. From table 1-2, It is revealed that MTSF, profit and availability decrease with the increase of failure rate $\left(\lambda_{1}\right)$ and maximum operation time ( $\alpha_{0}$ ), but the value of availability and MTSF increase with the increase of repair rate $(\theta)$ and preventive maintenance rate $(\alpha)$. The profit of the system declines with the increases of preventive maintenance rate $(\alpha)$. Thus finally it is concluded that a system in which priority to operation is given over preventive maintenance can be made more reliable and profitable to use

(i) By taking one more unit in cold standby.

(ii) By ignoring the concept of priority to operation over preventive maintenance.

(iii) By increasing the repair rate.

\section{REFERENCES}

[1] Gopalan, M.N. \& Nagarwall, H.E.: Cost-Benefit analysis of a one server two-unit cold standby system with repair and preventive maintenance, Microelectron Reliability, 25, 267-269, 1985.

[2] Gopalan, M.N. \& Bhanu, K.S.: Cost analysis of a twounit repairable system subject to online preventive maintenance and/ or repair, Microelectron Reliab., 35(2), 251-258, 1995.

[3] Malik, S.C.: Reliability models with priority for operation and repair with arrival time of server. Journal of Pure and Applied Mathematika Sciences, Vol. LXI (12), pp. 9-22, 2005.

[4] Malik, S.C., Nandal,P. \& Bhardwaj, R.K.: Economic analysis of repairable systems with random appearance and disappearance of the service facility. Journal of Mathematics and System Sciences, Vol. 3(1), pp. 36-49, 2007.

[5] Malik, S.C. and Kumar, A.: Reliability modeling of a computer system with priority to $\mathrm{s} / \mathrm{w}$ replacement over $\mathrm{h} / \mathrm{w}$ replacement subject to MOT and MRT, International Journal of Pure and Applied Mathematics, Vol. 3, No.9 (2012), pp.7191-7204. 
15. NUMERICAL ANALYSIS

\begin{tabular}{lllll}
\hline$\lambda_{1}$ & $\theta=0.2, \alpha_{0}=5$, & $\begin{array}{l}\theta=1.2, \alpha_{0}=5, \\
\alpha=2\end{array}$ & $\begin{array}{l}\theta=0.2, \alpha_{0}=5, \\
\alpha=5\end{array}$ & $\begin{array}{l}\theta=0.2, \alpha_{0}=10, \\
\alpha=2\end{array}$ \\
& & & & \\
\hline $\mathbf{0 . 0 1 0}$ & 139.4449 & 140.0669 & 195.6368 & 119.9094 \\
$\mathbf{0 . 0 1 1}$ & 126.7218 & 127.3396 & 177.4943 & 109.0912 \\
$\mathbf{0 . 0 1 2}$ & 116.1199 & 116.7336 & 162.3815 & 99.9112 \\
$\mathbf{0 . 0 1 3}$ & 107.1496 & 107.7593 & 149.5990 & 92.2198 \\
$\mathbf{0 . 0 1 4}$ & 99.4613 & 100.0670 & 138.6473 & 85.6272 \\
$\mathbf{0 . 0 1 5}$ & 92.7986 & 93.4003 & 129.1602 & 79.9138 \\
$\mathbf{0 . 0 1 6}$ & 86.9692 & 87.5670 & 120.8630 & 74.9146 \\
$\mathbf{0 . 0 1 7}$ & 81.8260 & 82.4200 & 113.5455 & 70.5037 \\
$\mathbf{0 . 0 1 8}$ & 77.2547 & 77.8448 & 107.0444 & 66.5829 \\
$\mathbf{0 . 0 1 9}$ & 73.1649 & 73.7513 & 101.2308 & 63.0750 \\
$\mathbf{0 . 0 2 0}$ & 69.4844 & 70.0671 & 96.0013 & 59.9179 \\
\hline
\end{tabular}

Table 1: MTSF vs. Failure Rate $\left(\lambda_{1}\right)$

\begin{tabular}{lcccc}
\hline$\lambda_{1}$ & $\theta=0.2$, & $\begin{array}{l}\theta=1.2, \alpha_{0}=5, \\
\alpha=2\end{array}$ & $\begin{array}{l}\theta=0.2, \alpha_{0}=5, \\
\alpha=5\end{array}$ & $\begin{array}{l}\theta=0.2, \alpha_{0}=10, \\
\alpha=2\end{array}$ \\
& $\alpha_{0}=5, \alpha=2$ & & & \\
\hline $\mathbf{0 . 0 1 0}$ & 0.9943 & 0.9964 & 0.9967 & 0.9937 \\
$\mathbf{0 . 0 1 1}$ & 0.9935 & 0.9960 & 0.9961 & 0.9928 \\
$\mathbf{0 . 0 1 2}$ & 0.9926 & 0.9957 & 0.9955 & 0.9920 \\
$\mathbf{0 . 0 1 3}$ & 0.9918 & 0.9953 & 0.9948 & 0.9910 \\
$\mathbf{0 . 0 1 4}$ & 0.9908 & 0.9950 & 0.9941 & 0.9901 \\
$\mathbf{0 . 0 1 5}$ & 0.9899 & 0.9946 & 0.9934 & 0.9891 \\
$\mathbf{0 . 0 1 6}$ & 0.9889 & 0.9942 & 0.9926 & 0.9881 \\
$\mathbf{0 . 0 1 7}$ & 0.9879 & 0.9939 & 0.9918 & 0.9870 \\
$\mathbf{0 . 0 1 8}$ & 0.9869 & 0.9935 & 0.9910 & 0.9859 \\
$\mathbf{0 . 0 1 9}$ & 0.9858 & 0.9931 & 0.9901 & 0.9848 \\
$\mathbf{0 . 0 2 0}$ & 0.9847 & 0.9928 & 0.9892 & 0.9836 \\
\hline
\end{tabular}

Table 2: Availability vs. Failure Rate $\left(\lambda_{1}\right)$

\begin{tabular}{lcccc}
\hline$\lambda_{1}$ & $\theta=0.2$, & $\begin{array}{l}\theta=1.2, \alpha_{0}=5, \\
\alpha=2\end{array}$ & $\begin{array}{l}\theta=0.2, \alpha_{0}=5, \\
\alpha=5\end{array}$ & $\begin{array}{l}\theta=0.2, \alpha_{0}=10, \\
\alpha=2\end{array}$ \\
& $\alpha_{0}=5, \alpha=2$ & & & \\
\hline $\mathbf{0 . 0 1 0}$ & 4.3945 & 4.4065 & 4.1417 & 4.3014 \\
$\mathbf{0 . 0 1 1}$ & 4.3900 & 4.4042 & 4.1396 & 4.2971 \\
$\mathbf{0 . 0 1 2}$ & 4.3853 & 4.4020 & 4.1374 & 4.2926 \\
$\mathbf{0 . 0 1 3}$ & 4.3804 & 4.3998 & 4.1349 & 4.2880 \\
$\mathbf{0 . 0 1 4}$ & 4.3754 & 4.3976 & 4.1323 & 4.2832 \\
$\mathbf{0 . 0 1 5}$ & 4.3701 & 4.3953 & 4.1295 & 4.2781 \\
$\mathbf{0 . 0 1 6}$ & 4.3647 & 4.3931 & 4.1264 & 4.2676 \\
$\mathbf{0 . 0 1 7}$ & 4.3592 & 4.3909 & 4.1232 & 4.2621 \\
$\mathbf{0 . 0 1 8}$ & 4.3534 & 4.3886 & 4.1198 & 4.2565 \\
$\mathbf{0 . 0 1 9}$ & 4.3475 & 4.3864 & 4.1163 & 4.2506 \\
$\mathbf{0 . 0 2 0}$ & 4.3415 & 4.3842 & 4.1125 & \\
\hline
\end{tabular}

Table 3: Profit vs. Failure Rate $\left(\lambda_{1}\right)$ 